\title{
Projections from the Ventral Respiratory Group to Phrenic and Intercostal Motoneurons in Cat: An Autoradiographic Study ${ }^{1}$
}

\author{
JACK L. FELDMAN, ${ }^{2}$ ARTHUR D. LOEWY, ${ }^{*}$ AND DEXTER F. SPECK ${ }^{3}$ \\ Departments of Physiology and Anesthesia, Northwestern University, Chicago, Illinois 60611 and *Department of Anatomy and \\ Neurobiology, Washington University, St. Louis, Missouri 63110
}

\begin{abstract}
Anterograde transport of tritiated amino acids (leucine, lysine, and proline) was used to examine the spinal projections of respiratory premotor neurons in the ventral respiratory group (VRG) of cats. This population of neurons corresponds anatomically with the nucleus ambiguus-retroambigualis. Small volumes (20 to $50 \mathrm{nl}$ ) of tritated amino acids were pressure ejected into the middle of the VRG through a micropipette which permitted simultaneous recording of respiratory modulated activity. In two cats injections were made caudal to the obex in regions which contained expiratory modulated neurons. In five cats injections were made rostral to the obex in regions containing inspiratory neurons. After a 2-week survival period, cats were anesthetized and perfused. The entire neuraxis was removed and processed using standard autoradiographic techniques. Transport of tritiated amino acids revealed a marked bilateral projection to lamina IX of the spinal cord at the C4 to $\mathrm{C} 6$ level and a primarily contralateral projection to laminae VIII and $I X$ in the thoracic spinal cord. Distinct descending pathways to the phrenic motor neurons were observed in the lateral funiculus and in the ventral funiculus; descending fibers to the intercostal motoneurons in the thoracic cord appeared to be restricted to the ventral funiculus. Labeling of axon terminals in both the cervical and thoracic cords was confined to ventral horn regions which contain motoneurons. These results suggest that monosynaptic projections from brainstem bulbospinal neurons to spinal motoneurons are important in controlling respiratory movements of the diaphragm and intercostal muscles.
\end{abstract}

The motoneurons innervating the major muscles of the respiratory pump in mammals are located in the mid-cervical and thoracic spinal

Received June 28, 1984: Revised November 12, 1984

Accepted November 12, 1984

${ }^{1}$ We would like to thank Donald R. McCrimmon and C. H. Price for their comments on the manuscript. This work was supported by National Institutes of Health Grant HL-23820. J. L. F. is a recipient of Research Career Development Award HL-00554, and D. F. S. is a recipient of National Research Service Award HL-06331. This work was also supported by Grantin-Aid 80723 from the American Heart Association, with funds contributed in part by the Missouri Heart Association and United States Public Health Service Grant HL-25449. A. D. L. is an Established Investigator of the American Heart Association.

${ }^{2}$ To whom correspondence should be sent.

${ }^{3}$ Present address: Department of Physiology and Biophysics, A. B. Chander Medical School, University of Kentucky, Lexington, KY 40536 cord. These neurons have rhythmic bursts of discharge that produce the alternating periods of inspiratory and expiratory muscle contraction and, ultimately, airflow. The respiratory periodicity exhibited by these neurons is due to descending inputs of brainstem origin (Eccles et al., 1962; Cohen et al., 1974). Two neuronal populations, the dorsal respiratory group (DRG) in the region of the ventrolateral nucleus of the tractus solitarius and the ventral respiratory group (VRG) in the region of the nucleus ambiguus-retroambigualis, have been identified by electrophysiological methods to contain bulbospinal neurons that provide a powerful paucisynaplic respiralory modulated drive to spinal motoneurons. Antidromic activation techniques show that at least $25 \%$ of the bulbospinal VRG neurons have axons that arborize in the region of the contralateral phrenic nucleus (Merrill, 1974). In fact, analysis of brainstem neuron $\rightarrow$ spinal respiratory nerve (motoneuron) cross-correlations (Cohen et al., 1974, Hilaire and Monteau, 1976; Feldman et al., 1983; McCrimmon et al., 1983) and intracellular spike-triggered averages (Fedorko et al., 1983; Lipski et al., 1983) indicate that these projections have a dominant monosynaptic component.

This study focuses on the VRG projections to the spinal cord. To determine precisely the target sites of descending projections from the VRG, we injected small volumes of tritiated amino acids into physiologically mapped subregions of the VRG in cats. Recording through the injection micropipette permitted precise placement of the injection within the VRG. This maximized the labeling of projections from VRG neurons and minimized labeling of non-respiratoryrelated projections arising from neurons in regions surrounding the VRG. The use of tritiated amino acids eliminated labeling due to transport by axons of passage through the injection site as occurs with horseradish peroxidase methods. Following standard protocols, the tissue was processed and examined for anterograde projections in the cervical and thoracic spinal cord.

\section{Materials and Methods}

In seven adult cats anesthesia was induced with ketamine hydrochloride $(0.4 \mathrm{ml}$, i.m.) which was followed by a mixture of chloralose and urethane (40 mg/kg and $250 \mathrm{mg} / \mathrm{kg}$, i.v., respectively). Cats were placed in an atraumatic headholder and an occipital craniotomy was made by rostral enlargement of the foramen magnum, exposing the dura covering the medulla for several millimeters around the obex. The dura was then cut, exposing the dorsal brainstem surface. A strain gauge connected to a differential amplifier was placed around the thorax to indicate phases of the respiratory cycle.

An amino acid mixture consisting of equal activities of tritiated leucine (specific activity, $110 \mathrm{Ci} / \mathrm{mmol}$ ), lysine $(70 \mathrm{Ci} / \mathrm{mmol})$, and proline $(100 \mathrm{Ci}$ ) mmol, New England Nuclear) was concentrated to $125 \mu \mathrm{Ci} / \mu \mathrm{l}$ in filtered saline (165 mM NaCl). A glass micropipette was filled from the tip to about 2 $\mathrm{cm}$ up the shank with silicone oil. About 3 to $5 \mu \mathrm{l}$ of the amino acid cocktail were then sucked into the tip by applying a moderate vacuum at the shank end of the electrode. A silver recording wire was inserted into the amino acid-saline solution in the electrode. The electrode was then sealed in a holder that connected the silver wire to a source follower for extracellular 
recording and the pipette to a solenoid valve-controlled pressure source to regulate pressure injection of the amino acid-saline solution.

In all experiments the targeted region of the VRG was initially mapped in the transverse plane by recording with a tungsten microelectrode. Injections were made in regions containing either predominantly inspiratory (I) or expiratory (E) modulated activity. In two cats initial mapping was done approximately $2 \mathrm{~mm}$ caudal to the obex in a region of the VRG where only expiratory modulated activity was recorded. In the other five cats, inspiratory modulated activity was mapped in the VRG from 1 to $4 \mathrm{~mm}$ rostral to the obex. After completion of the mapping, the glass micropipette was inserted into the brainstem and was targeted for the approximate center of (mapped) respiratory unit activity. During the descent of the glass micropipette, neuronal activity was monitored. If the distance below the dorsal surface of the brainstem (3300 to $4700 \mu \mathrm{m}$ ) and the range over which we could record inspiratory neuronal activity $(\sim 700$ to $1000 \mu \mathrm{m})$ suggested that we were indeed on target, the electrode was positioned at the depth corresponding to the center of recorded respiratory activity. While monitoring the micropipette meniscus through a $\times 100$ microscope with a calibrated reticule, 5 to $15 \mathrm{nl}$ of amino acid-saline solution were ejected. These small injections were repeated every 5 to $10 \mathrm{~min}$ until a total volume of 20 to $50 \mathrm{nl}$ was reached. Ten minutes after the last injection, the micropipette was slowly removed.

Gelfoam was placed over the exposed brainstem and the overlying muscle and skin were sutured. Cats were allowed to recover and received daily prophylactic doses of broad spectrum antibiotics. Within 7? $\mathrm{hr}$ after the surgery, cats began to eat and appeared healthy and alert. After 2 weeks, animals were re-anesthetized as previously described. The cats were then perfused transcardially with 1 liter of warm hoparinized saline (1000 units, $40^{\circ} \mathrm{C}$ ) which was followed by 2 liters of $10 \%$ formalin at room temperature. The entire neuraxis was removed.

Tissue was processed usirig standard procedures for autoradiography (Cowan et al., 1972). Fifty-micrometer frozen sections were cut. Every tenth section was mounted on slides and dipped in nuclear emulsion. Exposure times averaged 10 weeks after which the slides were developed. Sections were counterstained with thionin and coverslipped.

Injection sites and labeled terminals were reconstructed with a microscope equipped with a drawing tube which superimposed the images of the tissue section and a bit pad cursor. Silver grains were readily observed under darkfield illumination. By visual examination at a magnification of $\times 75$, regions containing concentrations of silver grains above background counts were identified. For each section, the position of these grains was entered into a computer file via the bit pad. Typically, labeling of axons, terminal axonal arborizations, and synapses from 8 to 24 consecutive tissue sections were superimposed. With the aid of computer software, each $\times 75$ reconstruction was reduced in size and superimposed on a typical spinal cord segment drawn at $\times 20$. Thus, we were able to obtain precise positioning information for an entire transverse section, even in instances where spatial localization was not obvious at the level of a single section.

\section{Results}

All injections were centered in the VRG, as determined by both physiological mapping and subsequent anatomical identification. Injection sites ranged from $2 \mathrm{~mm}$ caudal to the obex to approximately $5 \mathrm{~mm}$ rostral to the obex. At all sites the neuronal discharge pattern was respiratory modulated. The respiratory modulated activity was predominantly expiratory in two cats and inspiratory in five cats. Only neurons with respiratory modulated spiking patterns were recorded within 350 to $500 \mu \mathrm{m}$ of the injection site (e.g., Figs. 1 and 2). From reconstruction of the penetrations made with the tungsten electrode prior to injection, we estimate that the mediolateral extent of the population extended \pm 400 to $600 \mu \mathrm{m}$ from the injection site.

Figure 1 is a complete reconstruction of the label observed in the spinal cord after injection into an inspiratory modulated region of the VRG rostral to the obex. Figure 2 shows a similar reconstruction after injection into an expiratory modulated region of the VRG caudal to the obex. The specific patterns of label observed in all seven cats are represented by these two examples and will be discussed in detail.

\section{Axonal arbors and terminal synaptic fields in spinal cord gray matter}

C1 to C3. In four cases, we did not observe any concentration of label in the rostral cervical segments (Fig. 1). In three cases, silver grains were found in laminae VII to IX (Fig. 2). Two of these cats also had bilateral labeling of the intermediomedialis nucleus.

$\mathrm{C} 4$ to $\mathrm{C}$. The major concentration of labeled terminals was located bilaterally in the nucleus lateromedialis of lamina IX of the ventral horn (Rexed, 1952) (Figs. 3 and 4, left). The label was uniformly distributed throughout this region, which forms a rostralcaudal column from $\mathrm{C} 4$ to $\mathrm{C} 6$ consisting of aggregates of cells separated by regions with few cells. This region is the unique location of phrenic motoneurons (Webber et al., 1979; Rikard-Bell and Bystrzycka, 1980; Goshgarian and Rafols, 1981). The center of the labeled clusters was approximately 1.2 to $1.4 \mathrm{~mm}$ lateral to the midline in all segments. A slight (100 to $200 \mu \mathrm{m}$ ) lateral displacement of the cell column and associated label was seen in the ventral horn enlargement at C6 (Fig. 3).

The density of labeling in lamina IX was greater in sections that contained many cell somata than in sections with few or no cell somata. Label was also found in regions both medial and lateral to lamina IX (laminae VII and VIII). This label did not seem to be associated with somata of neurons outside lamina IX, instead, it appeared to radiate toward or from lamina IX (Figs. 3 and $4 A$ ). Occasionally patterns of label suggestive of individual axons were observed crossing the midline veritral to the central canal. In no case did the dorsal and intermediate parts of the C4 to C6 gray matter (laminae I to $\mathrm{VI}$ ) show any concentration of label above background levels.

In all seven cats, the contralateral label was noticeably denser than that on the ipsilateral side, and the heaviest labeling was observed in the C5 spinal segment (Figs. 1 to 3 ). In experiments with inspiratory modulated injection sites, labeling of lamina IX in the contralateral $\mathrm{C} 4$ to $\mathrm{C} 6$ segments was considerably denser than the terminal labeling seen in the thoracic spinal cord. However, in the two cats injected caudal to the obex, the density of thoracic and cervical labeling did not differ as much.

C7 and C8. In three of four cases in which there was no labeling of $\mathrm{C} 1$ to $\mathrm{C} 3$, no concentration of label in the caudal cervical segments was found. In the remaining four cats, silver grains in C7 and C8 were seen concentrated in the region of lamina IX. Label was most dense on the contralateral side in three of these cats; label was evenly distributed in the fourth cat.

T1 to T12. In the three cats with label in $\mathrm{C} 1$ to $\mathrm{C} 3$ and $\mathrm{C} 7$ and $\mathrm{C8}$, there was also obvious labeling of the interomediolateral cell column (ML) from T1 to T6. This label was most donsc on the ipsilateral side, although it was sometimes observed bilaterally. In all seven cats the major concentration of label was restricted to the ventral horn on the side contralateral to the injection. Silver grains were found in close proximity to large neurons in laminae VIII and IX (Fig. 4, right). In many single sections there was a seemingly diffuse distribution of label in the ventral horn. Superposition of label from 10 to 25 serial sections revealed a striking clustering of label which coincided with the concentrations of ventral horn neurons (see Figs. 1,2 , and 4). With injections rostral to the obex in inspiratory modulated regions, the density of this thoracic label was less than that observed surrounding C4 to C6 lamina IX motoneurons (Figs. 1 and 4). Injections caudal to the obex still resulted in dense labeling in the vicinity of $\mathrm{C} 4$ to $\mathrm{C} 6$ motoneurons, although the apparent ratio of labeling in the C5 segment compared to that seen in the thoracic ventral horn was not as great.

$\angle 1$ to $L 3$. Labeling of terminals in these segments was observed in the two experiments involving injection sites caudal to the obex. Terminal label was again localized to laminae VIII and IX (Fig. 2).

\section{Bulbospinal axons}

The axons of neurons at the injection site descended bilaterally in both the lateral and ventral funiculi (Figs. 1 and 2). In the upper cervical segments ( $\mathrm{C} 1$ and $\mathrm{C} 2$ ), the labeling of these two tracts appeared approximately equal. The density of labeling in the lateral funiculus began to decrease at the $\mathrm{C} 3$ to $\mathrm{C} 4$ spinal segments and was almost nonexistent after C5. (In two cats labeling of the ipsilateral 


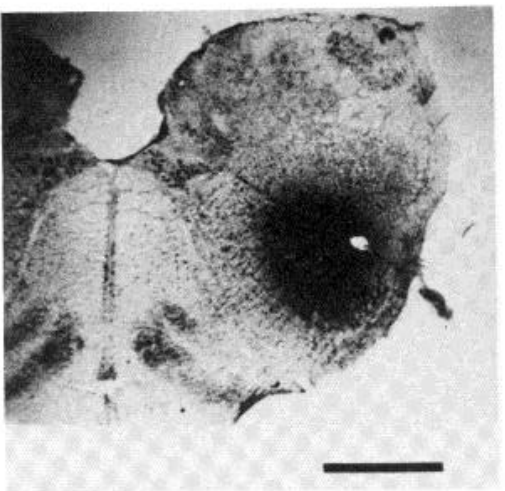

$+383$
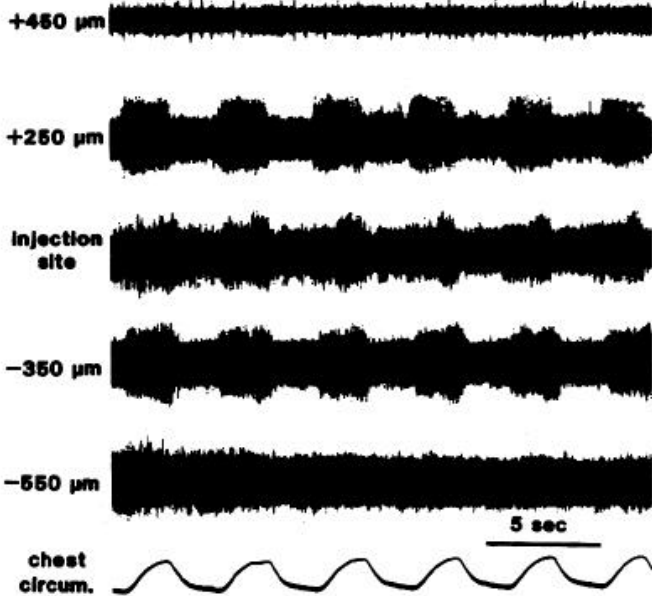

B

C1

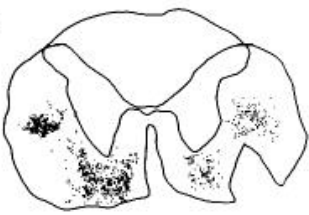

C2

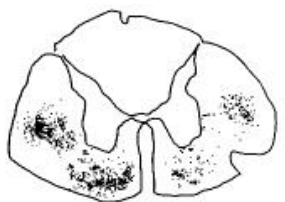

с3

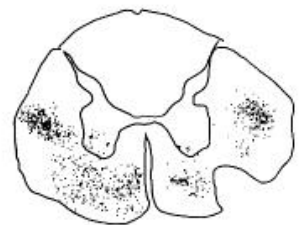

C4

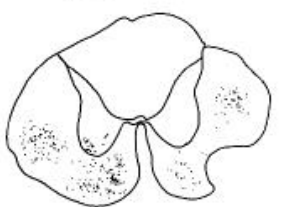

C5

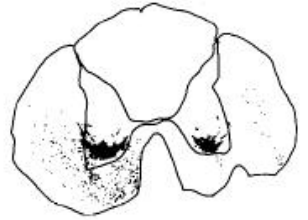

C6

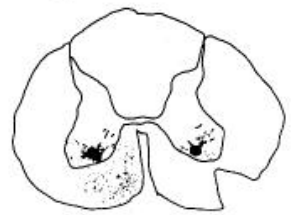

C7

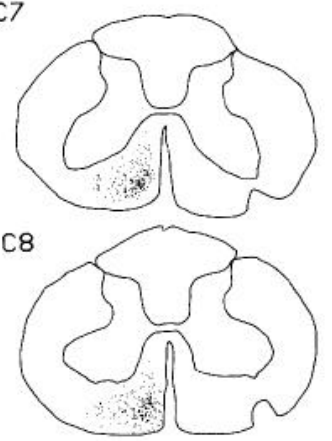

T1

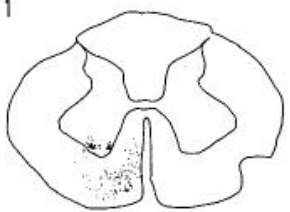

T2

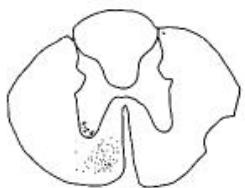

T3

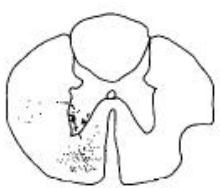

T4

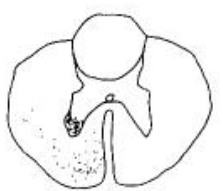

T5

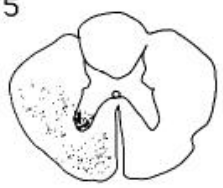

T6

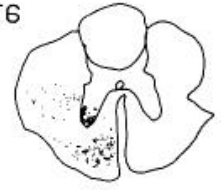

T7

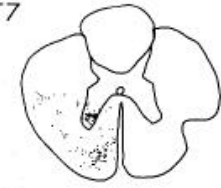

T8

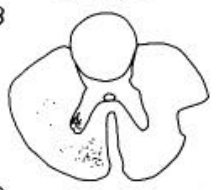

TS

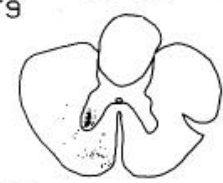

T10

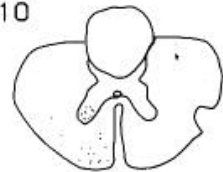

T11

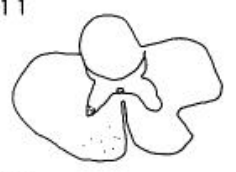

T12

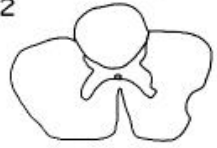

Figure 1. Cat 383. Distribution of descending projections observed with autoradiography after injection of tritiated amino acids into an inspiratory modulated region of the VRG. A, Left: Photomicrograph of the injection site approximately $2.0 \mathrm{~mm}$ rostral to the obex. Bar, $2 \mathrm{~mm}$. Right: Inspiratory modulated activity recorded around the injection site. $B$, Computer-aided reconstructions of the label seen in frontal sections of the spinal cord. Dots represent the presence of silver grains. Each drawing is a composite of the label observed in 24 tissue sections at the spinal level indicated. Labeling was most dense on the contralateral (left) side. See Figures 3 and 4 for details of the projections to C4, C5, C6, and T2. 
A
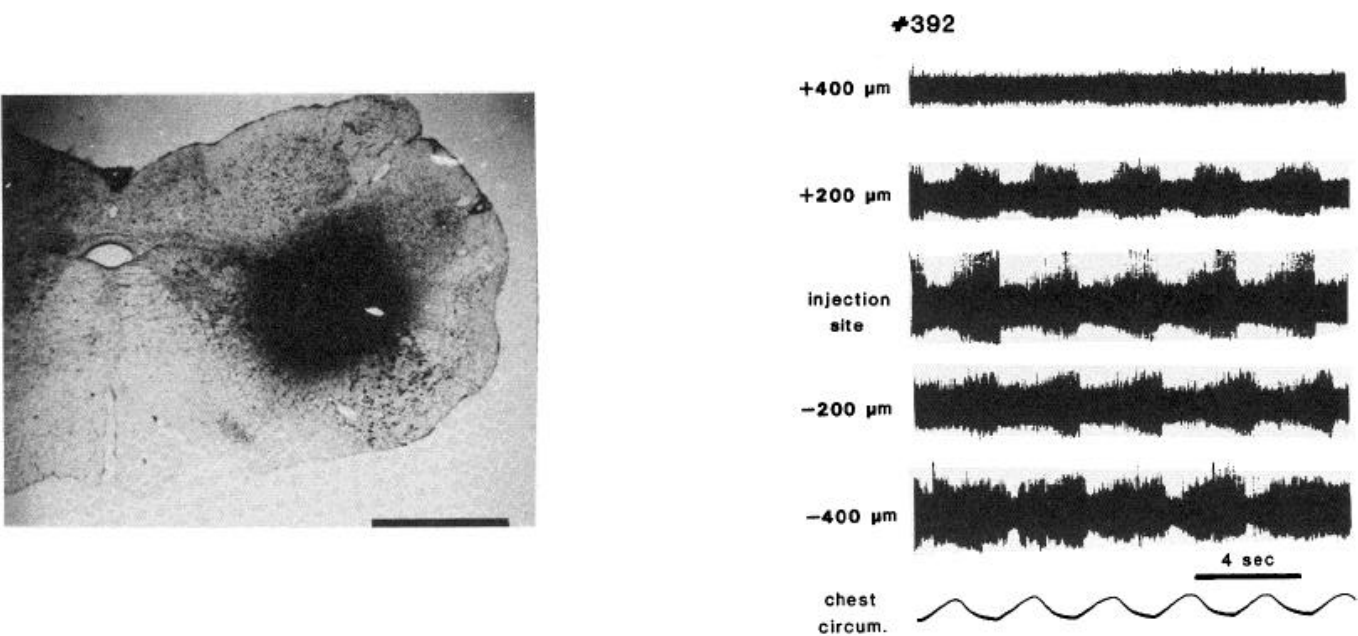

B
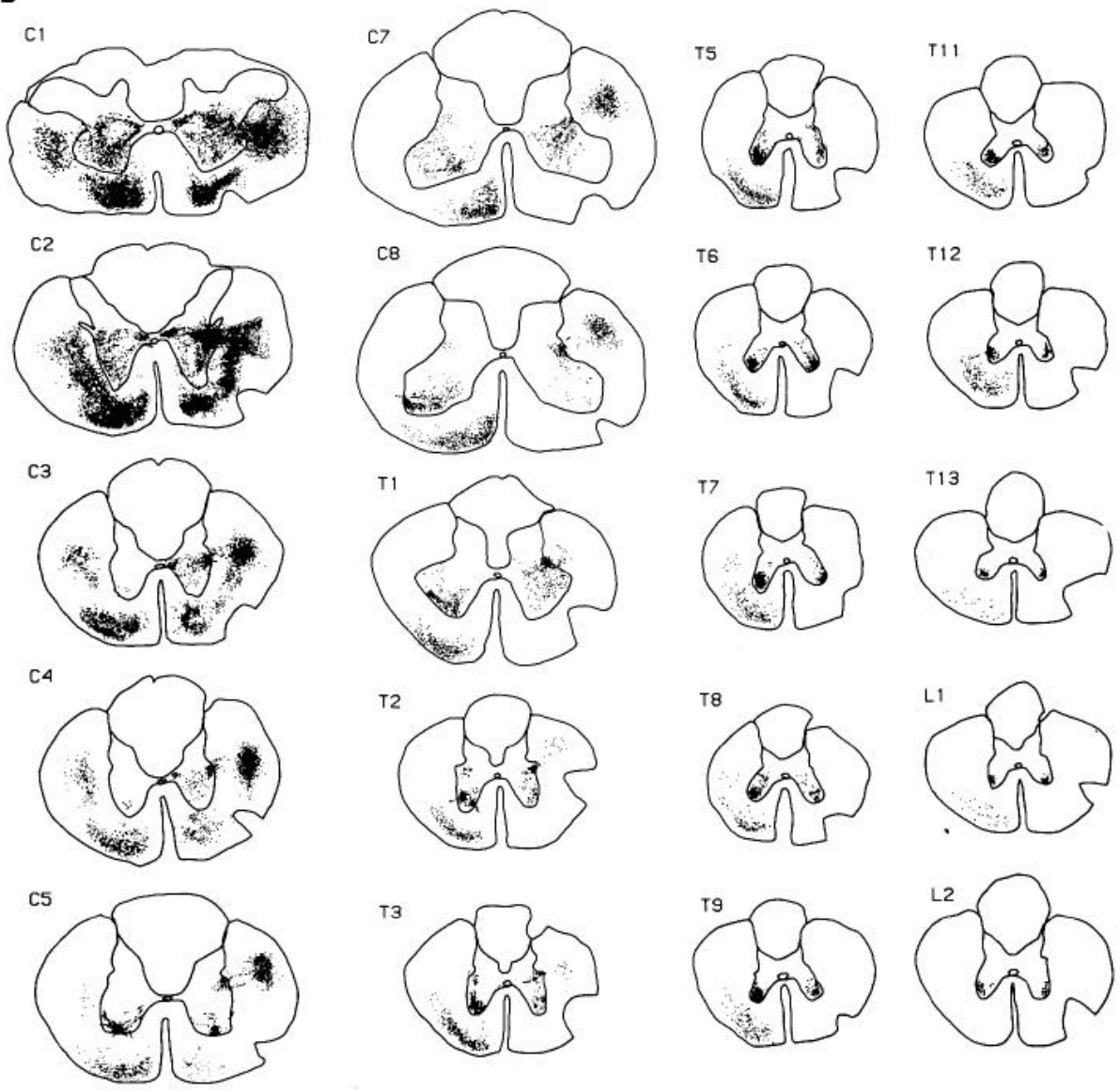

T3
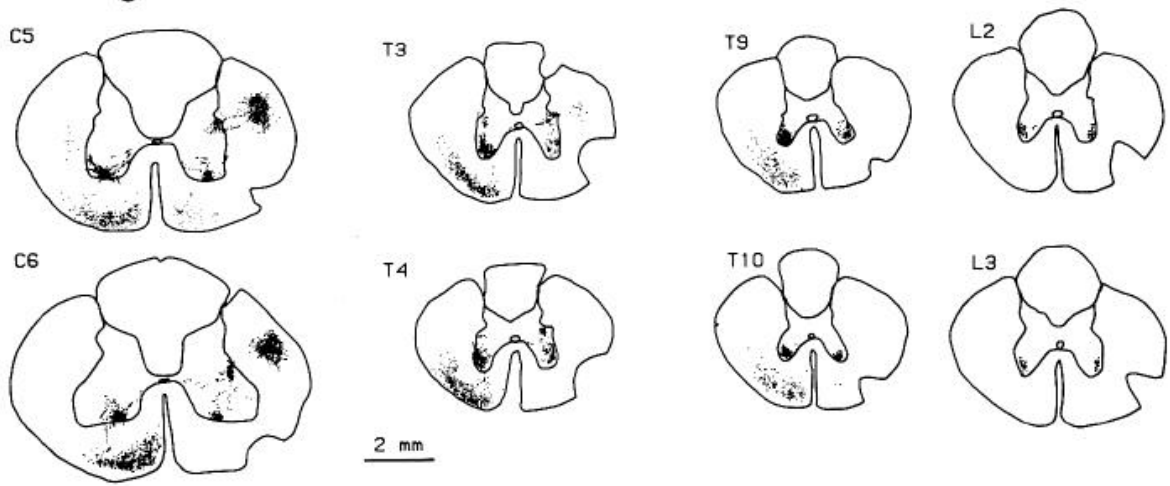

T4
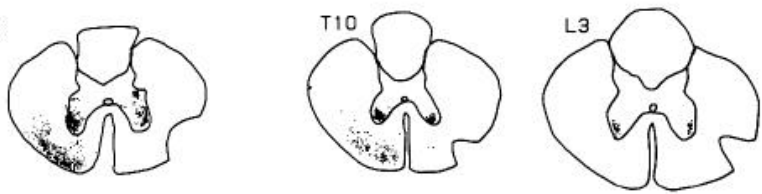

Figure 2. Cat 392. Distribution of descending projections observed with autoradiography after injection of tritiated amino acids into an expiratory modulated region of the VRG. A, Left: Photomicrograph of the injection site approximately $2.0 \mathrm{~mm}$ caudal to the obex. Bar, 2 mm. Right: Expiratory modulated activity recorded around the injection site. $B$, Computer-aided reconstructions of the label seen in frontal sections. Sections are represented as in Figure 1. 


\section{C4}
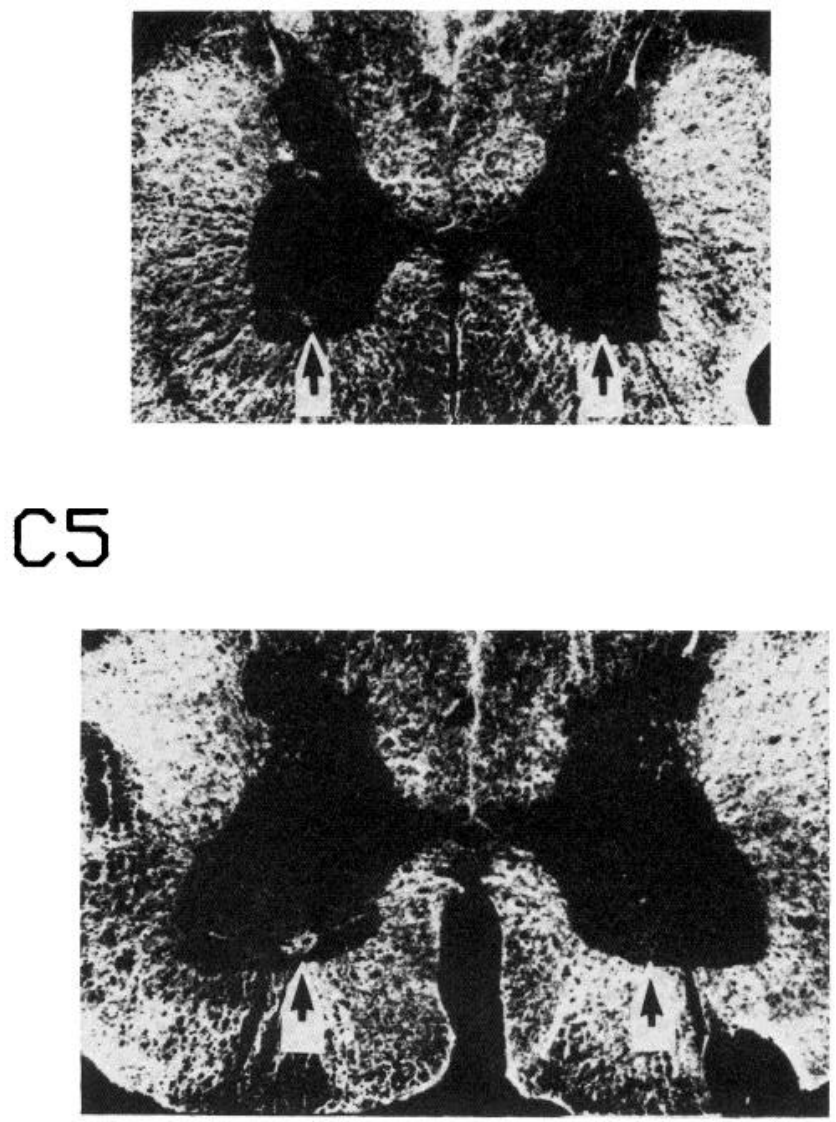

\section{C6}

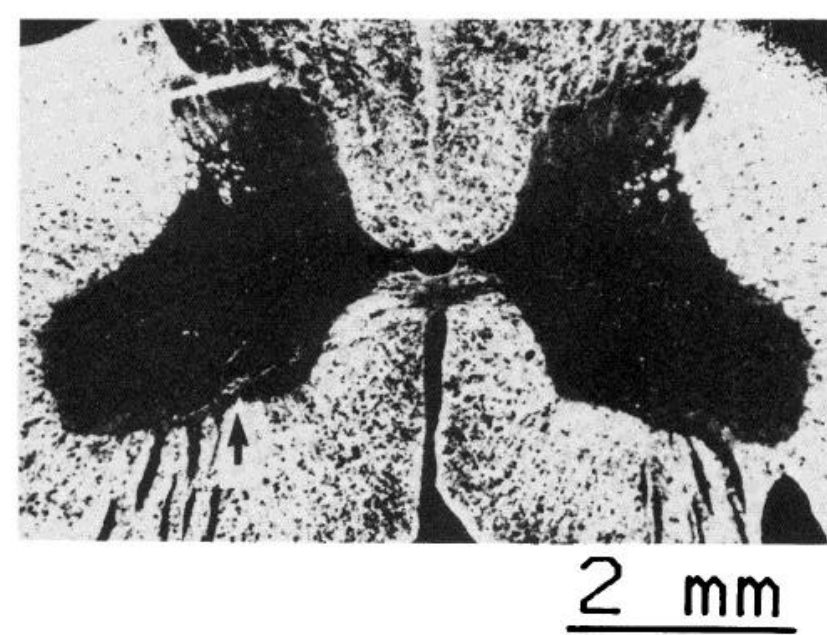

Figure 3. Darkfield photomicrographs of VRG projections to the gray matter in representative sections of $\mathrm{C} 4, \mathrm{C} 5$, and $\mathrm{C} 6$ from cat 746 . Injection was in the right VRG $2 \mathrm{~mm}$ rostral to the obex. The projection to the lateromedialis nuclei of lamina IX is indicated by the arrows. Note that the labeling is heaviest on the contralateral (left) side in the C5 segment. lateral funiculus continued into thoracic segments where it appeared to terminate in the $\mathrm{ML}$.) Similarly, the projection in the ventral funiculus diminished bilaterally in C4 to C6 segments. Caudal to C6, labeled axons were restricted primarily to the contralateral ventral funiculus. This tract dissipated as it traveled through the thoracic cord. With VRG injections rostral to the obex, this tract was no longer apparent by T12. With VRG injections caudal to the obex, label in the ventral funiculus was found in the upper lumbar cord (L1 to L3).

\section{Discussion}

In previous anatomical studies of the descending projections of the nucleus ambiguus-retroambigualis, tritiated amino acids were injected into brainstem regions determined by stereotaxic methods (Martin et al., 1981; Holstege and Kuypers, 1982). These authors were restricted to post hoc statements concerning the physiological behavior of the neurons in the region of the injection site. In the present study, we recorded from the pipette containing the amino acid cocktail; this enabled injection of amino acids into the center of the regions of neurons with spontaneous inspiratory or expiratory activity in the VRG. Since the injections were of small volume and were centered in the respiratory neuronal regions, the number of non-respiratory modulated neurons outside these regions that transported amino acids was minimized. Prior studies which used stereotaxic placement of tritiated amino acids in the region of the VRG report substantial labeling of the IML. In four cases in this study, only slight or no labeling of the IML was observed (e.g., Fig. 1). In three cases, however, significant labeling of the IML was observed (e.g., Fig. 2). A recent study employing retrograde transport of horseradish peroxidase (Caverson et al., 1983) indicated that the neurons projecting to the IML in the second thoracic segment are located medial and ventral to the VRG; very few cells were labeled in the nucleus ambiguus-retroambigualis at the level of the obex (see also Gebber and Barman, 1984). Thus, our observation of a more selective projection in several cases than that reported by other authors may be due to the more precise targeting of our injections. We think that a significant component of the additional label observed in two cats (Fig. 2) results from transport by bulbospinal sympathetic neurons located outside the region of respiratory modulated neuronal activity. However, other factors may also account for the observed differences. For example, longer survival times (Holstege and Kuypers, 1982) would allow time for more amino acid to be transported, perhaps resulting in detectable label in more regions. Subtle differences in experimental procedures or in the protocols for processing autoradiographs may also contribute to these differences.

In neuroanatomical experiments one must consider at least two caveats. ( $i$ ) The region of active uptake of the amino acids is impossible to circumscribe; it is likely that neurons in regions adjacent to those identified as containing respiratory neurons transported the amino acids. (ii) Quiescent neuronș within the regions containing respiratory modulated neurons could also transport the amino acids; some of these neurons may have functions unrelated to respiratory control. Since both of these possibilities could produce false positive results concerning respiratory-related projections, it is striking how limited the terminal labeling was in these experiments.

Bulbospinal pathways. The bulbospinal projections from inspiratory neurons in cat are usually described as contralateral projections in the ventrolateral funiculus (Newsom Davis and Plum, 1972), although the existence of an ipsilateral descending projection is acknowledged (Cohen, 1979; von Euler, 1983). Electrophysiological studies suggest that 10 to $15 \%$ of the axons may descend on the ipsilateral side (Merrill, 1974; Sessle et al., 1978). However, neuroanatomical studies have suggested a stronger bilateral projection from the inspiratory neurons in the DRG (Loewy and Burton, 1978). In this study the projection from the VRG to the phrenic motor nucleus was primarily contralateral, although there was also a significant ipsilateral projection (Figs. 1 and 3). 


\section{C5}

\section{T2}
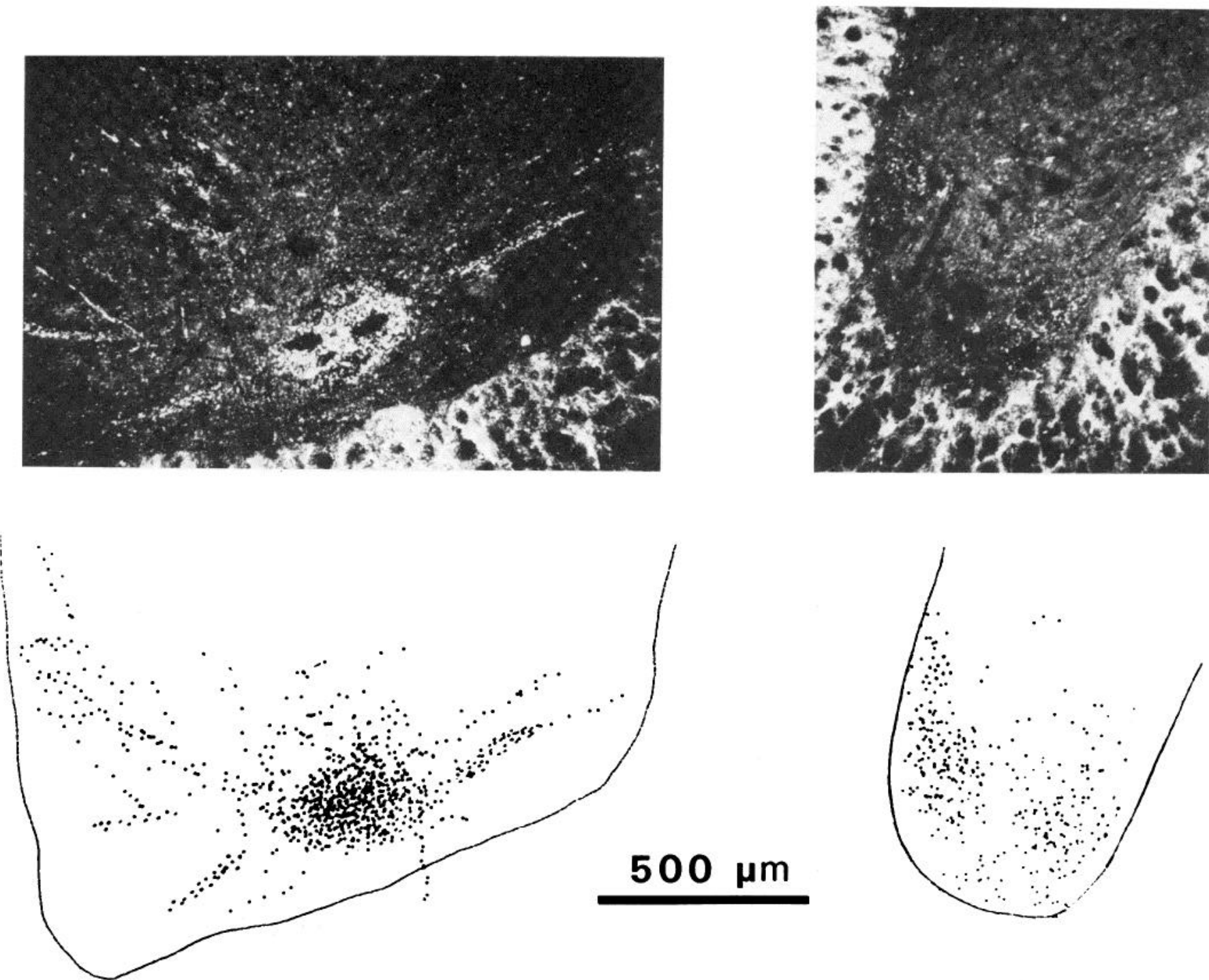

Figure 4. Darkfield photomicrographs of VRG projections to the contralateral ventral horn in a cervical (C5) and thoracic (T2) segment from cat 746. Below each photomicrograph is a reconstruction of the label drawn from 12 consecutive sections.

Several investigators have localized the descending inspiratory pathway to the ventrolateral funiculus; however, others have published data which suggest a different location. Torvik (1957) concluded that most of the solitariospinal fibers descend in the lateral funiculus. Similarly, Kuypers and Maisky (1977) found that axons of solitary tract neurons descend through both the lateral and the ventral funiculi, while the fibers arising from neurons in the nucleus retroambigualis descend in the ventral and ventrolateral funiculi. In a recent study from this laboratory (Feldman et al., 1984), a site of C2 spinal cord stimulation which elicited maximal orthodromic phrenic nerve activity was found in the lateral funiculus. Stimulation at this site also antidromically activated approximately $33 \%$ of the inspiratory neurons in both the DRG and the VRG. The results of the present study suggest that many VRG inspiratory neurons have axons which descend in the lateral funiculus and terminate in the phrenic nucleus, since this lateral projection diminishes significantly in the $\mathrm{C} 4$ to $\mathrm{C} 6$ region. The ventral funiculus contains axons of VRG neurons which project to intercostal motoneurons and may project to phrenic motoneurons.
Projections to phrenic motoneurons. Although the DRG is considered a major source of descending inspiratory drive to the phrenic motor nucleus (Cohen et al., 1974; Hilaire and Monteau, 1976; Fedorko et al., 1983), it has also been demonstrated that VRG neurons project to phrenic motoneurons. Correlation analysis of the timing of VRG neuronal impulse activity with whole phrenic nerve activity (Hilaire and Monteau, 1976; Feldman and Speck, 1983; McCrimmon et al., 1983) indicates that approximately $40 \%$ of the VRG inspiratory neurons make mono- or paucisynaptic projections to phrenic motoneurons. This interpretation has been challenged by Fedorko et al. (1983) based on their results from spike-triggered averages of VRG neuronal impulse activity and membrane potential recordings from single phrenic motoneurons. These authors suggest that only 2 to $7 \%$ of the VRG neurons make monosynaptic projections to phrenic motoneurons. They conclude that the majority of interactions indicated by extracellular correlation analysis must be due to multisynaptic projections. This implies that a significant component of the projection is via interneurons. Since in this study no terminal fields in regions outside the phrenic nucleus were labeled 
in three cats, it is unlikely that spinal interneurons are important in this projection. Of course, the relevant premotor interneuron could be within the phrenic motor nucleus, but as discussed below, this does not seem likely. The apparent disparity between the results of Fedorko et al. (1983) and those of the present study could be due to several factors. (i) Amino acids are likely to be transported by all neurons in the vicinity of the injection site, whereas extracellular recording experiments would only examine a limited subset of active neurons. Thus, the anatomical studies might given an overestimate of the strength of the projection to any given area relative to the number of normally active cells. (ii) Anatomical techniques might identify projections of neurons in or around the injection site that do not participate in descending respiratory drive commands, e.g., sympathetic prepreganglionic neurons (Caverson et al., 1983; Gebber and Barman, 1984). The combination of respiratory neuronal network architecture and the use of small injections in physiologically identified regions reduced the magnitude of this contounding factor. (iii) In experiments involving recording from a limited number of pairs of cells, there could be sampling problems that would bias the results. For example, the strength of projections from individual VRG neurons to individual phrenic motoneurons may be limited. This limitation could be structural or it could vary with physiological state, as has been hypothesized to occur with branch point failure (Luscher et al., 1982). Thus, the likelihood of demonstrating a monosynaptic connection between any bulbospinal neuron projecting to a subset of phrenic motoneurons and a single phrenic motoneuron (or even several proximal ones) would be limited and would depend on fortuitous sampling. Nevertheless, a cross-correlation analysis between this single VRG neuron and whole phrenic norvo activity should reveal a monosynaptic projection.

Phrenic motoneurons which provide the efferent innervation of the diaphragm via the phrenic nerve are unambiguously confined to the nucleus lateromedialis of lamina IX in cervical segments C4 to C6 of the cat (Duron et al., 1979; Webber et al., 1979; Rikard-Bell and Bystrzycka, 1980; Goshgarian and Rafols, 1981). There is circumstantial evidence that the phrenic motor nucleus may contain some interneurons. Keswani et al. (1954) reported interneurons in lamina IX based on chromatolysis studies. Also, Hilaire et al. (1983a) have obtained recordings from two interneurons in the vicinity of phrenic motoneurons which were likely to be Renshaw cells, excited by collaterals of phrenic motoneurons. From counts of both horseradish peroxidase-labeled and unlabeled nucleolated cells within lamina IX, Webber et al. (1979) suggest that there may be interneurons within this area. Conspicuously absent, however, it any direct evidence of (excitatory) interneurons in the phrenic motor nucleus. Regardless, it is apparent that the phrenic motor neurons contains predominantly motoneurons. When recording intracellularly from neurons in lamina $\mathrm{IX}$, all neurons with respiratory modulated oscillations of membrane potential can be activated antidromically with phrenic nerve stimulation (Webber and Pleshka, 1981; Cameron et al., 1983; but see Hilaire et al., 1983a).

The distribution of silver grains in the region of the phrenic motor nucleus corresponds with the location of the cell somas and dendrites. Cameron et al. (1983) have observed that the dendrites of phrenic motoneurons project rostrocaudally within the nucleus, dorsomedially to cross the midline, and both laterally and dorsolaterally into the lateral funiculus. They also observed a small number of ventrally directed dendrites; however, no dendrites were found to project dorsally. As seen in Figures 1 to 3 , the distribution of descending projections and axon terminals from the VRG bulbospinal neurons overlaps all three of these dendritic fields. Note that no label was detected immediately dorsal to the phrenic nucleus.

Projections to intercostal motoneurons. The short-term synchronization observed between thoracic intercostal motoneurons is thought to arise from the descending projections of bulbospinal respiratory neurons (Kirkwood et al., 1982). Cross-correlation studies have demonstrated monosynaptic projections from VRG inspiratory neurons to external intercostal motoneurons (Hilaire and Monteau, 1976; McCrimmon et al., 1983).

The motoneurons innervating the intercostal muscles are located in laminae VIII and IX of the thoracic spinal cord. Clusters of motoneurons are distributed throughout two overlapping areas. The lateral cluster (nucleus lateromedialis thoracalis) innervates primarily the parasternal intercostal muscles, although a small percentage of external and internal intercostal motoneurons are also located within this region. The medial area (nucleus ventromedialis) contains motoneurons which project to paravertebral, external, and internal intercostal muscles (Larnicol et al., 1982). The paravertebral motoneurons are concentrated in the most medial portion of this nucleus and have recently been demonstrated to have a strong respiratory periodicity (Hilaire et al., 1983b). It has been postulated that the dual role the intercostal muscles play in respiration and posture is reflected in the relative degree of respiratory modulation in each group of muscles (parasterrial, paravertebral, and lateral) (Larnicol et al., 1982). Since the respiratory periodicity in the intercostal musculature is greatest in the parasternal and paravertebral muscles, the descending respiratory inputs to the thoracic spinal cord might be concentrated in the lateral and medial aspects of the ventral horn. The distribution of label throughout lamina IX seen in this study is consistent with this suggestion.

Significance of monosynaptic projections to respiratory motoneurons. An important observations of this study is the predominant confinement of cervical and thoracic spinal cord projections to ventral horn regions containing motoneurons innervating the diaphragm and intercostal muscles. At the cervical level, the label was clustered in the region of the phrenic motoneuronal somata and dendrites. At the thoracic level, the label was clustered in the region of intercostal motoneurons. In three cases, we did not detect concentrations of silver grains indicative of terminal fields outside these regions, with the noted exceptions of the $\mathrm{ML}$.

The almost exlcusive $\mathrm{C} 4$ to $\mathrm{C} 6$ projection to the phrenic motor nucleus (as well as to the T1 to T12 intercostal motor nuclei) seen in these studies strongly suggests a monosynaptic projection from the brainstem premotor neurons to these motoncurons. In most limb motor systems (e.g., locomotion), the supraspinal input to the spinal motoneurons is mediated by spinal motoneurons which are important in the integration of afferent input with the descending command. These interneurons are commonly located in laminae $V$ to $V I I$ (Nyberg-Hansen, 1965). Thus, in limb motor systems, motoneuronal discharge produces movements that activate afferents in the limb. These afferents enter the spinal cord in segments proximal to the motoneurons and premotor interneurons and thus can make direct contact with the spinal interneurons which also receive descending commands. In contrast, respiratory movements activate both somatic and visceral, e.g., pulmonary stretch receptor, afferents. The latter enter the central nervous system via cranial nerves and are especially important in the control of respiratory timing and pattern. In the brainstem these afferents can make monosynaptic connections with the interneurons which conduct the descending commands directly to respiratory motoneu ons. Although both limb and respiratory muscles utilize interneuror $s$ to integrate the centrally generated pattern with afferent feedbc.ck, these systems differ in the location of premotor neurons (spina cord versus brainstem) and in the length of the premotor neuronal axons.

Recognizing the numerous caveats, we interpret our results to indicate that the predominant projections from VRG bulbospinal neurons are direct to phrenic and intercostal motoneurons and are likely to be monosynaptic. These bulbospinal respiratory neurons are unlikely to be involved in the generation of respiratory rhythm (Feldman et al., 1984) and therefore must be driven by the mechanisms which are responsible for respiratory rhythmogenesis. In addition to rhythmic inputs, many of the VRG bulbospinal neurons receive inputs from peripheral respiratory afferents (Cohen et al., 1974; Sessle et al., 1978). These facts suggest that the VRG neurons may integrate both central inspiratory drive and afferent inputs to 
produce the appropriate descending command for the precise coordinated inspiratory motoneuronal discharge necessary for ventilation in the cat.

\section{References}

Cameron, W. E., D. B. Averill, and A. J. Berger (1983) Morphology of cat phrenic motoneurons as revealed by intracellular injection of horseradish peroxidase. J. Comp. Neurol. 219: 70-80.

Caverson, M. M., J. Ciriello, and F. R. Calaresu (1983) Direct pathway from cardiovascular neurons in the ventrolateral medulla to the region of the intermediolateral nucleus of the upper thoracic cord: An anatomical and electrophysiological investigation in the cat. J. Auton. Nerv. Syst. 9: 451475.

Cohen, M. I. (1979) Neturogenesis of respiratory rhythm in the mammal. Physiol. Rev. 59: 1105-1173.

Cohen, M. I., M. F. Piercey, P. M. Gootman, and P. Wolotsky (1974) Synaptic connections between medullary inspiratory nourons and phrenic motonou rons as revealed by cross-correlation. Brain Res. 81: 319-324

Cowan, W. M., D. I. Gootlieb, A. E. Hendrickson, J. L. Price, and T. A. Woolsey (1972) The autoradiographic demonstration of axonal connections in the central nervous sytem. Brain Res. 37: 21-51.

Duron, B., D. Marlot, N. Larnicol, M. C. Jung-Caillol, and J. M. Macron (1979) Somatotopy in the phrenic motor nucleus of the cat as revealed by retrograde transport of horseradish peroxidase. Neurosci. Lett. 14: 159163.

Eccles, R. M. T. A. Sears, and C. N. Shealy (1962) Intracellular recording from respiratory motoneurons of the thoracic spinal cord of the cat. Nature 193: 844-846

Fedorko, L., E. G. Merrill, and J. Lipski (1983) Two descending medullary inspiratory pathways to phrenic motoneurons. Neurosci. Lett. 43: 285291.

Feldman, J. L., and D. F. Speck (1983) interactions among inspiratory neurons in dorsal and ventral respiratory groups in cat medulla. J. Neurophysiol. 49: 472-490.

Feldman, J. L., D. R. McCrimmon, and D. F. Speck (1984) Effect of synchronous activation of medullary inspiratory bulbo-spinal neurones on phrenic nerve discharge in cat. J. Physiol. (Lond.) 347: 241-254.

Gebber, G. L., and S. M. Barman (1984) Electrophysiology of neurons in cat ventrolateral medulla projecting to intermediolateral sympathetic nucleus. Soc. Neurosci. Abstr. 10: 33 .

Goshgarian, H. G., and J. A. Rafols (1981) The phrenic nucleus of the albino rat: A correlative HRP and Golgi study. J. Comp. Neurol. 201: 441-456.

Hilaire, G., and R. Monteau (1976) Connexions entre les neurones inspiratoire bulbaires et les motoneurones phreniques et intercostaux. J. Physiol (Paris) 72: 987-1000

Hilaire, G., M. Khatib, and R. Monteau (1983a) Spontaneous respiratory activity of phrenic and intercostal Renshaw cells. Neurosci. Lett. 43: $97-$ 101

Hilaire, G., J. G. Nicholls, and T. A. Sears (1983b) Central and proprioceptive influences on the activity of levator costae motoneurones in the cat. $J$. Physiol. (Lond.) 342: 527-548.

Holstege, G., and H. G. J. M. Kuypers (1982) The anatomy of brain stem pathways to the spinal cord in cat. A labeled amino acid tracing study. Prog. Brain Res. 57: 145-175.

Keswani, N. H., R. A. Groat, and W. H. Hollinshead (1954) Localization of the phrenic nucleus in the spinal cord of the cat. J. Anat. Soc. (India) 3: 82-89.

Kirkwood, P. A., T. A. Sears, D. L. Tuck, and R. H. Westgaard (1982) Variations in the time course of the synchronization of intercostal motoneurones in the cat. J. Physiol. (Lond.) 327: 105-135.

Kuypers, H. G. J. M., and V. A. Maisky (1977) Funicular trajectories of descending brain stem pathways in cat. Brain Res. 136: 159-165.

Larnicol, N., D. Rose, D. Marlot, and B. Duron (1982) Spinal localization of the intercostal motoneurones innervating the upper thoracic spaces. Neurosci. Lett. 31: 13-18.

Lipski, J., L. Kubin, and J. Jodkowski (1983) Synaptic action of R Beta neurons on phrenic motoneurons studied with spike-triggered averaging. Brain Res. 288: 105-118.

Loewy, A. D., and H. Burton (1978) Nuclei of the solitary tract: Efferent projections to the lower brainstem and spinal cord of the cat. J. Comp. Neurol. 181: 421-450.

Luscher, H. R., E. Henneman, and J. Mathis (1982) Transmission failure at la synapses on cat spinal motoneurons. Neuroscience (Suppl.) 7: 135.

Martin, G. F., T. Cabana, A. O. Humbertson, Jr., L. C. Laxson, and W. M. Panneton (1981) Spinal projections from the medullary reticular formation of the North American opossum: Evidence for connectional heterogeneity. J. Comp. Neurol. 196: 663-682.

McCrimmon, D. R., D. F. Speck, and J. L. Feldman (1983) Projections of medullary inspiratory neurons to phrenic and intercostal motor nerves assessed by cross-correlation analysis. Soc. Neurosci. Abstr. 9: 362.

Merrill, E. G. (1974) Finding a respiratory function for the medullary respiratory ncurons. In Essays on the Nervous System, R. Bellairs and E. G. Gray, eds., pp. 451-486, Clarendon Press, Oxford.

Newsom Davis, J., and F. Plum (1972) Separation of descending spinal pall ways lo respiratory motoneurons. Exp. Neurol. 34: 78-94.

Nyberg-Hansen, R. (1965) Sites and mode of termination of reticulo-spinal fibers in the cat. An experimental study with silver impregnation techniques. J. Comp. Neurol. 124: 71-100.

Rexed, B. (1952) The cytoarchitectonic organization of the spinal cord in the cat. J. Comp. Neurol. 96: 415-496.

Rikard-Bell, G. C., and E. K. Bystrzycka (1980) Localization of phrenic motor nucleus in the cat and rabbit studied with horseradish peroxidase. Brain Res. 194: 479-483.

Sessle, B. J., L. F. Greenwood, J. P. Lund, and G. E. Lucier (1978) Effects of upper respiratory tract stimuli on respiration and single respiratory neurons in the adult cat. Exp. Neurol. 61: 245-259.

Torvik, A. (1957) The spinal projection from the nucleus of the solitary tract. An experimental study in the cat. J. Anat. 91: 314-322.

von Euler, C. (1983) On the contral pattcrn gencrator for the basic breathing rhythmicity. J. Appl. Physiol. 55: 1647-1659.

Webber, C. L., Jr., and K. Pleschka (1981) Central respiratory drive potentials and membrane potential trajectories in phrenic motoneurons. Brain Res. 211: 179-184.

Webber, C. L., Jr., R. D. Wurster, and J. M. Chung (1979) Cat phrenic nucleus architecture as revealed by horseradish peroxidase mapping. Exp. Brain Res. 35: 395-406. 